\title{
RELIABILITY ASSESSMENT OF AN ISOLATED HYBRID MICROGRID USING MARKOV MODELING AND MONTE CARLO SIMULATION
}

\author{
Ahmad F. Saleem ${ }^{1}$ - Mohammad AlMuhaini ${ }^{1 *}$ \\ ${ }^{1}$ Electrical Engineering Department, King Fahd University of Petroleum and Minerals, Dhahran, Saudi Arabia
}

\begin{tabular}{l} 
ARTICLE INFO \\
\hline Article history: \\
Received: 5.6 .2019$. \\
Received in revised form: 16.4 .2020$. \\
Accepted: 17.4 .2020$. \\
\hline Keywords: \\
Photovoltaic \\
Wind Turbine Generator \\
Micro-Gas Turbine \\
Monte Carlo Simulation \\
Markov Modeling \\
\hline DOI: https://doi.org/10.30765/er.1478
\end{tabular}

\begin{abstract}
:
The importance of conducting adequacy assessments of standalone systems with integrated renewable generation sources is growing. In this work, the focus is placed on the reliability assessment of an isolated microgrid operating on renewable energy generated by wind turbines (WTs) and photovoltaic (PV) panels. Batteries for storage were included in the model because of their crucial role in the system's feasibility. Additional microgas turbines (MGTs) served as conventional backup. The sequential Monte Carlo simulation (SMCS) method was used to carry out simulations of the system, which was modeled using Markov matrices. Input data, such as wind speed, solar irradiance, and ambient air temperature, were used to simulate the power outputs of the generators. These historical data were fitted into appropriate distributions to extract corresponding parameters when simulating essential key factors necessary to produce the renewable power generation models. The adequacy model of the MGTs was obtained by employing the two-state reliability model, which was also superimposed with the generation models of WTs, PV panels, and batteries. The IEEE Roy Billinton test system (RBTS) was used for demand modelling. Common reliability indices were computed, and the system availability margins were evaluated.
\end{abstract}

\section{Introduction}

Constantly changing loads and a growing number of customers and their geographical dispersal are some of the challenges currently facing power utilities. Traditional modes of power system growth are limited by environmental and economic policies. Integrating renewable energy generators locally can aid in overcoming these issues. Reliability of energy generation using renewable sources has been a subject of research since the early 1980s. The electrical power output of photovoltaic (PV) and wind systems, along with some conventional generation sources such as micro-gas turbines (MGTs) and diesel generators, were modeled in [1]. Strategies for attaining higher reliability levels in standalone hybrid power systems were discussed in [2]. Cost effects and reliability of an isolated small power system were evaluated in [3] by Billinton and colleagues.

The authors computed several composite system indices using the Monte Carlo simulation (MCS) technique. In [4], a model was similarly developed and simulated using sequential Monte Carlo simulation (SMCS) to calculate the reliability indices of a microgrid system.

The focus of this work is placed on testing an isolated microgrid comprised of green energy generators, such as PV panels, wind turbine generators (WTGs), and conventional MGTs, to calculate the system's viability. A two-state model was used to model MGTs. The same approach was employed in combination with power curve characteristics to model the PV and WTG systems. Storage media is essential in such

\footnotetext{
${ }^{*}$ Corresponding author

E-mail address: muhaini@kfupm.edu.sa
} 
systems; in this work, this was realized using a battery model. The developed generation models were combined with a storage model to supply the IEEE Roy Billinton test system (RBTS) demand model [5, 6]. The system's availability margins, and reliability indices were computed using MCS and Markov modeling techniques with the goal of employing a probabilistic approach instead of a deterministic one. A sensitivity analysis was also conducted to study the effects of various sizing configurations of the generation and storage models.

Sections 2.1, 2.2, and 2.3 of this article describe the development of the power output models of the WT, $\mathrm{PV}$, and MGT systems, respectively. The demand model is presented in Section 6, which is combined with the generation models described in the previous sections to build the storage model explained in Section 2.4. Section 3 provides an overview of the essential techniques for modeling the system's availability and calculating the reliability indices, including the MCS method and the Markov matrices. Section 4 presents a case study conducted to evaluate system availability using SMCS and Markov modeling. A sensitivity analysis is also presented in this section. Section 5 presents the conclusions that can be derived from the results and offers some recommendations for future research directions.

\section{System Modeling}

\subsection{Wind System Model}

Wind energy is an intermittent renewable resource, making it difficult to accurately forecast power production at any given point in time. Nonetheless, the percentage of time during which diverse power levels would be available can be computed. The expected proportions of time during which WT will be required to meet specific demand levels, the frequency with which backup power will be needed, and other planning aspects must be determined in order to increase system reliability.

The main factor for electrical power output in WTGs is wind velocity. In general, wind speed can be divided into three levels: cut-in, rated, and cut-out speeds. The cut-in speed is the lower bound for wind speed, below which power generation is impossible, and thus the WT is switched off. If the velocity is between the rated and cut-in velocity limits, variable output power is generated. If the velocity exceeds the rated speed but is below the cut-out level, the WT generates constant output power equal to its rated value. To prevent the WT from surpassing the mechanical safety bounds, it also shuts off if the wind velocity exceeds the rated cut-off speed. The wind speed-power output characteristic based on these relationships is illustrated in Figure 1, and a simplified model is given in Eq. (1):

$$
P w(v)=\left\{\begin{array}{cc}
0 & 0 \leq v \leq V_{c i} \\
P_{\text {rated }} \cdot \frac{v-V_{c i}}{v-V_{c i}} & V_{c i} \leq v \leq V_{r} \\
P_{\text {rated }} & V_{r} \leq v \leq V_{c o} \\
0 & V_{c o} \leq v
\end{array}\right.
$$

where $P_{\text {rated }}$ and $V_{r}$ are the rated power and velocity, respectively; $V_{c i}$ and $V_{c o}$ are the cut-in and cut-out wind speed, respectively; and $v$ denotes the wind velocity.

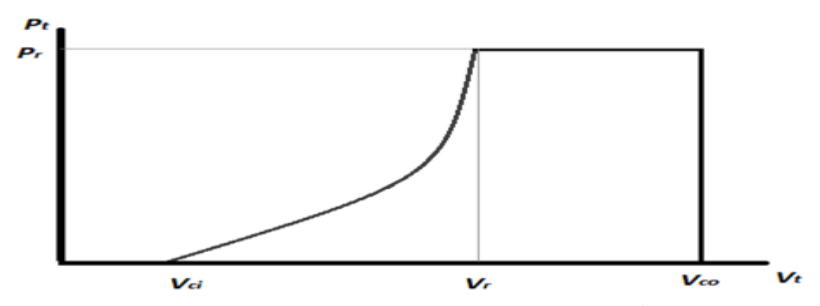

Figure 1. Power curve of a typical WT.

Wind velocity and direction change continuously, leading to variations in the power generated by the WTs. Owing to this uncertainty, wind speed behavior could be simulated using a probabilistic approach 
whereby the data are sampled in hourly intervals. A Weibull distribution is suitable for modeling the wind velocity probability distribution using the probability density function:

$$
F(v)=\left(\frac{\beta}{\alpha^{\beta}}\right) v^{\beta-1} \exp \left[-\left(\frac{v}{\alpha}\right)^{\beta}\right]
$$

where $\alpha$ and $\beta$ are the scale and shape parameters describing the Weibull distribution.

The steps involved in adopting one of the commonly used techniques for simulating wind velocity using historical data are presented below.

- Step 1: Create a uniformly distributed random variable $U$ with values in the $[0,1]$ range.

- Step 2: Calculate the parameters $\alpha$ and $\beta$ of the Weibull distribution using Eq. (3):

$$
\alpha=\frac{v}{\Gamma}\left(1+\frac{1}{\beta}\right) \quad \beta=\left(\frac{\sigma}{v}\right)^{-1.086}
$$

where the wind speed's standard deviation is denoted by $\sigma$, and $\Gamma$ represents the gamma function.

- Step 3: Compute $v$ by means of the inverse transform method of the Weibull distribution:

$$
v=\alpha(-\ln U)^{1 / \beta}
$$

The Weibull distribution of the wind velocity parameter is sensitive to any variations in the $\alpha$ and $\beta$ parameter values. Consequently, it is essential to obtain the wind speed data pertaining to a specific area for several years in order to account for all seasons. The WT rated values of $P_{\text {rated }}, V_{r}, V_{c i}$, and $V_{c o}$ used in this study are listed in Table 1.

Table 1. WT parameters.

\begin{tabular}{cccc}
\hline \hline $\mathrm{P}_{\text {rated }}(\mathrm{W})$ & $\mathrm{V}_{\mathrm{r}}(\mathrm{m} / \mathrm{s})$ & $\mathrm{V}_{\mathrm{ci}}(\mathrm{m} / \mathrm{s})$ & $\mathrm{V}_{\mathrm{co}}(\mathrm{m} / \mathrm{s})$ \\
\hline 1500 & 12 & 3.5 & 14 \\
\hline \hline
\end{tabular}

\subsection{Photovoltaic System Model}

PV cells generate direct current during daytime hours. This electric power is either consumed immediately to meet the demand or can be reserved for future consumption. When simulating such energy resources, it is essential to obtain sufficient solar data corresponding to the location under investigation. Solar irradiance and air ambient temperature are atmospheric data that play a central role in PV power generation. Due to the uncertainty inherent in this data, a procedure similar to that used to generate wind input data for WTs must be applied to the collected historical PV data. Weather conditions vary with seasons. Therefore, a suitable probability distribution function should be used when fitting the gathered data to determine the scale and shape parameters for both ambient temperature and solar irradiance.

The current and voltage characteristic given in Equation (5) are essential for PV characterization, as they also incorporate ambient temperature $T_{A}$ and solar irradiance $s$.

$$
\begin{gathered}
T_{C}=T_{A}+\frac{s\left(N_{O T}-20\right)}{.8} \\
I=s\left[I_{S C}+K_{I}\left(T_{C}-25\right)\right] \\
V=V_{O C}-K_{V} T_{C}
\end{gathered}
$$

where $T_{C}$ refers to the PV cell temperature (in ${ }^{\circ} \mathrm{C}$ ), $I_{S C}$ represents the short circuit current of the cell (in A), $K_{I}$ refers to the temperature constant of the short circuit current (in $\mathrm{A} /{ }^{\circ} \mathrm{C}$ ), $V_{O C}$ stands for the open circuit 
voltage (in V), and $K_{V}$ denotes the temperature factor corresponding to the open circuit voltage $\left(\mathrm{V} /{ }^{\circ} \mathrm{C}\right)$. The values of those constants are provided by the PV manufacturer in the data sheet. In addition to the PV power output evaluation, the Fill Factor $(F F)$ was also computed using the expression below:

$$
F F=\left(V_{M P P} I_{M P P}\right) /\left(V_{O C} I_{S C}\right.
$$

where $V_{M P P}$ and $I_{M P P}$ denote the maximum power point voltage and current, respectively. The electrical power generated by a PV panel consisting of $N$ modules can be obtained as follows:

$$
P_{P V}=N * F F * V * I
$$

A PV panel with 10 cells per panel was used in this study. All relevant parameters are given in Table 2.

\begin{tabular}{|c|c|c|c|c|c|c|}
\hline $\begin{array}{l}\mathrm{I}_{\mathrm{SC}} \\
(\mathrm{A})\end{array}$ & $\begin{array}{l}\mathrm{V}_{\mathrm{OC}} \\
(\mathrm{V})\end{array}$ & $\begin{array}{c}\mathrm{V}_{\mathrm{mpp}} \\
(\mathrm{V})\end{array}$ & $\begin{array}{l}I_{\text {mpp }} \\
(\mathrm{A})\end{array}$ & $\begin{array}{c}\mathrm{K}_{\mathrm{I}} \\
(\%)\end{array}$ & $\begin{array}{c}\mathrm{K}_{\mathrm{V}} \\
(\%)\end{array}$ & $\begin{array}{l}\mathrm{N}_{\mathrm{OT}} \\
\left({ }^{\circ} \mathrm{C}\right)\end{array}$ \\
\hline 8.682 & 36.9 & 30.01 & 8.011 & 0.0717 & -0.401 & 47 \\
\hline
\end{tabular}

Table 2. PV parameters.

\subsection{MGT System Model}

MGT engines generate electrical power by extracting energy from burning fuel in a combustion chamber. In this arrangement, the fast-flowing burning output gases drive the turbine. MGTs are commonly used in distribution networks and sometimes serve as backup distributed generators (DGs). Gas is a relatively reliable energy source as its use results in fewer failures during operation, and its output is considered predictable. Thus, modeling the system power output is simple, as it primarily depends on the operational hours. In this work, the binary model shown in Figure 2 was used to model the MGTs. It was then combined with the renewable generation and storage models, discussed later. The failure and repair times of the various components were simulated by performing the following steps:

- Step 1: Create two uniformly distributed random variables $U$ and $U^{\prime}$ with values in the $[0,1]$ range.

- Step 2: Evaluate TTF and TTR using the expressions below:

$$
\begin{aligned}
& T T F=-M M T F \cdot \ln U \\
& T T R=-M T T R \cdot \ln U^{\prime}
\end{aligned}
$$

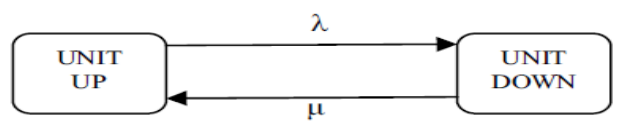

Figure 2. Binary model applicable to $M G$.

The parameters listed in Table 3 were used to evaluate the output power of MGTs that serve as standby energy supply or backup for the standalone system in case the renewable power and energy stored cannot meet the demand.

Table 3. MGT parameters.

\begin{tabular}{cccc}
\hline \hline Rated Power $(\mathrm{W})$ & Minimum Power $(\mathrm{W})$ & MTTF $(\mathrm{h})$ & MTTR $(\mathrm{h})$ \\
\hline 10000 & 250 & 7500 & 100 \\
\hline \hline
\end{tabular}

\subsection{Storage (Battery) Model}

Conventional energy sources such as fossil fuels and gas can be easily transported and stored. This is not the case for most renewable forms of energy because they are rather sporadic, making their availability 
highly uncertain. Consequently, batteries are a commonly used energy storage medium when renewable power generation is abundant. The surplus of energy after supplying the demand are stored for later use when production of renewable energy is limited.

A battery model was designed in [9], where the battery energy storage was limited by the maximum power charge/discharge rates along with the maximum capacity. The model was based on the assumption that when renewable power generation exceeds the demand, the battery charges until it reaches its maximum capacity. Similarly, when the supply cannot meet the demand, the battery discharges until it becomes empty. Figure 3 illustrates an overview of the algorithm developed to model the battery handling process, while Table 4 provides pertinent nomenclature.

Table 4. Nomenclature used in Figure 3.

\begin{tabular}{lc}
\hline \hline Power surplus at time instant $i$ & $\operatorname{Surp}(i)$ \\
Maximum power charging rate & $P c_{\max }$ \\
Maximum power discharging rate & $P d_{\max }$ \\
Actual charging/discharging rate & $P c$ \\
Rated battery capacity & $E_{\max }$ \\
Battery energy state of charge & $E_{b a t}(i)$ \\
Previous time instant state of charge & $E_{b a t}(i-1)$ \\
Hourly time step & $d t$ \\
\hline
\end{tabular}

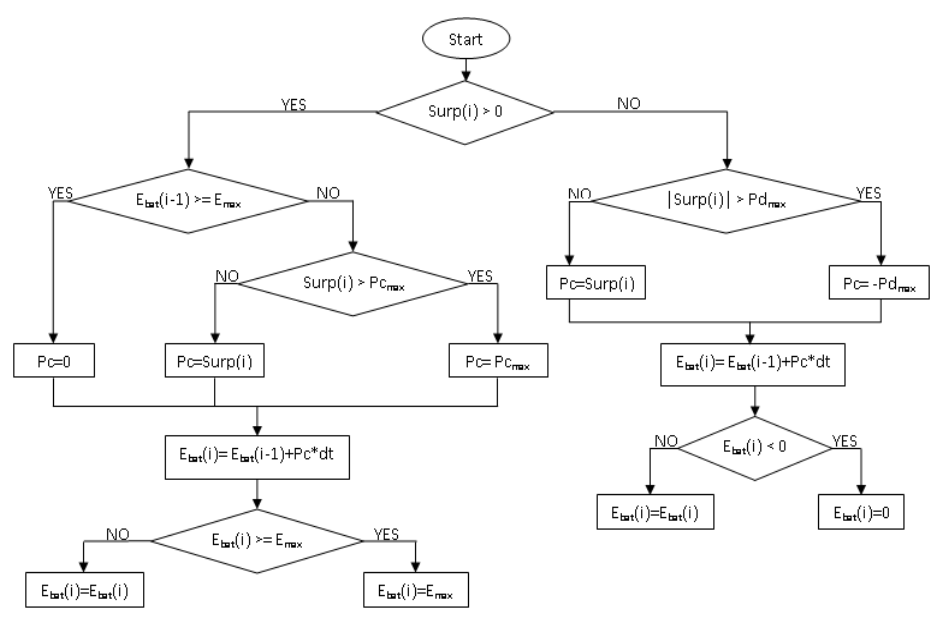

Figure 3. Battery model.

\subsection{Load Model}

The well-known RBTS was used as a demand model in this work to evaluate the isolated system reliability. Load behavior is primarily influenced by seasonal events and weather conditions, which tend to occur periodically throughout the year. Thus, historical data can be used to model the load. To develop a RBTS demand model and construct the load profile, hourly, daily, and monthly weighting factors were adopted. A section of a typical annual residential load curve is shown in Figure 4. Equation (9) was used to calculate the load points at specified times.

$$
L(t)=P_{w} P_{d} P_{h}(t)
$$




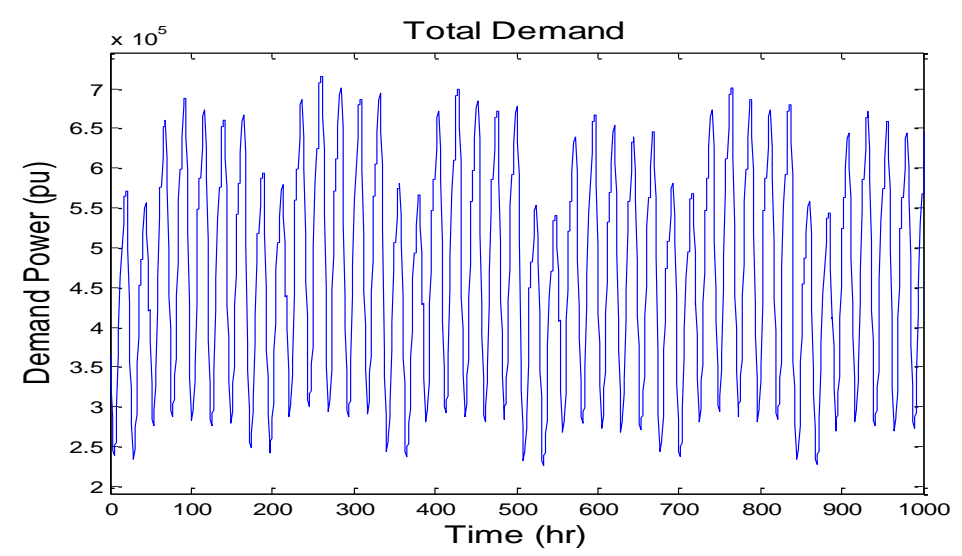

Figure 4. RBTS annual residential load curve.

\section{Microgrid Adequacy Assessment}

\subsection{MCS Technique}

MCS can generally be employed to define any method that uses statistical sampling to estimate solutions to quantitative problems. This approach allows for the exploration of the behavior of sophisticated systems using random variables as input parameters. MCS can be utilized to simulate failures and, due to the fact that they occur randomly in power systems, predict the behavior of system components. The time sequential MCS is applied when the system's behavior is dependent on previous events, which was the case in this study.

MCS has been extensively used for diverse applications. For example, analytical expressions were employed in [7] for evaluating reliability indices to calculate their expectations and variances in order to obtain their probability density functions.

The authors of [8] studied microgrids with the aim of optimizing renewable generation capacities. The goal was to reduce total generation costs and unserved power quantities. Optimization was conducted through the particle swarm optimization technique, while MCS was employed in iterative generation reliability studies.

\subsection{Setting the Network Topology}

The standalone microgrid was modeled using a simple single bus topology. Consequently, all DGs, batteries, and loads were assumed to be connected to a single point of common coupling, as depicted in Figure 5. In addition, the following assumptions were made:

1) Buses, cables, and power electronic devices in the system are $100 \%$ reliable, thus resulting in no power losses.

2) No power losses arise from the battery storage system.

In this study, one hour was chosen as the minimum time step for simulating weather conditions.

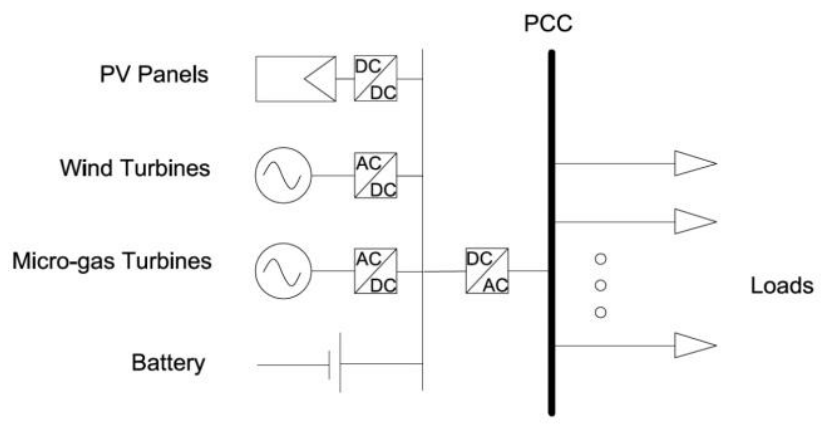

Figure 5. System network topology. 


\subsection{Attaining Availability Power Margins}

The previously described procedure was used in simulating the generation, storage, and demand models. Next, the power surplus was obtained in order to determine the system availability margins. A positive surplus indicates that the renewable generation system can satisfy the demand, whereas its negative value implies that the unmet demand must be supplied by the batteries. If the energy stored in the batteries is insufficient, the conventional backup units become operational. A failure occurs when Eq. (10) is true.

$$
\text { Renewable generation }+ \text { Battery storage }+(\text { MGT power if used })<\text { Demand }
$$

Based on the 1-hour temporal resolution, the overall number of failures is equal to the number of hours the system was unavailable. The system availability is then calculated using:

$$
\begin{aligned}
\text { Unavailability }= & \text { No. of failures per year } \times d t / \text { No. of hours per year } \\
& \text { Availability }=1-\text { Unavailability }
\end{aligned}
$$

\subsection{Reliability Indices Evaluation}

For the $i$ th sampled year, the loss of load duration $\left(L L D_{i}\right)$ measured in hours and the energy not supplied $\left(E N S_{i}\right)$ in $\mathrm{kWh}$ were obtained by observing the hourly power margin. The reliability indices pertaining to $N$ years were therefore estimated using the following expressions. The loss of load expectation (LOLE) in hours/year was measured using:

Loss of Load Expectation (LOLE), in hours/year:

$$
L O L E=\frac{\sum_{i=1}^{N} L L D_{i}}{N} \quad(h r / y r)
$$

Loss of Energy Expectation (LOEE):

$$
L O E E=\frac{\sum_{i=1}^{N} E N S_{i}}{N} \quad(k W h / \text { year })
$$

Average Energy Not Supplied per Interruption (AENSI):

$$
\text { AENSI }=\frac{\text { Total Energy Not Supplied }}{\text { Total Customer Interruptions }} \quad(\text { W/inter. })
$$

DG Supply during Interruptions (DGSI) (used with MGTs):

$$
D G S I=\frac{D G \text { Power Output }}{\text { Energy Not Supplied }}=\frac{\sum P_{D G, i}}{E N S} \quad(W / W h)
$$

\subsection{Markov Modelling of the System}

The aim of the present study was to develop a Markov model of the system simulated using the SMCS technique. Thus, the states that would best characterize the system were defined as follows:

- State 1 (S1): Total renewable generation > Demand

- State 2 (S2): Total renewable generation + Energy stored in batteries $>$ Demand

- State 3 (S3): Total renewable generation + Energy stored in batteries + Energy from MGTs $>$ Demand

- State 4 (S4): Total renewable generation + Energy stored in batteries + Energy from MGTs < Demand

These four states represent the system availability and unavailability under different circumstances. In order to calculate the availability power margins using Markov modeling, the system's transition rates needed to be computed first. The state transition matrix ( $\sigma$-matrix) shown in Eq. (16) contains the rates of departure between the different states. 


$$
\sigma=\left[\begin{array}{cccc}
0 & \sigma_{12} & \cdots & \sigma_{1 n} \\
\sigma_{21} & 0 & \cdots & \sigma_{2 n} \\
\vdots & \vdots & \ddots & \vdots \\
\sigma_{n 1} & \sigma_{n 2} & \cdots & 0
\end{array}\right]
$$

where $\sigma_{i j}$ is the departure rate from state $i$ to state $j$. This rate can be calculated as the ratio of the total number of departures from state $i$ to state $j$ and the total number of instances the system has been in state $i$.

In this context, the $Q$-matrix was used to form the coefficient matrix of the Markov differential equations by transposing the elements in the $\sigma$-matrix and replacing the diagonal elements by a negative sum of all elements in the same column, as shown in Eq. (17) below.

$$
Q=\left[\begin{array}{cccc}
-\sum_{j=2}^{n} \sigma_{1 j} & \sigma_{21} & \cdots & \sigma_{n 1} \\
\sigma_{12} & -\sum_{\substack{j=1 \\
j \neq 2}}^{n} \sigma_{2 j} & \cdots & \sigma_{n 2} \\
\vdots & \vdots & \ddots & \vdots \\
\sigma_{1 n} & \sigma_{2 n} & \cdots & -\sum_{j=1}^{n-1} \sigma_{n j}
\end{array}\right]
$$

After forming the $Q$-matrix, the Markovian set of differential equations was formed, as shown below.

$$
\left[\begin{array}{cccc}
-\sum_{j=2}^{n} \sigma_{1 j} & \sigma_{21} & \cdots & \sigma_{n 1} \\
\sigma_{12} & -\sum_{j=1}^{n} \sigma_{2 j} & \cdots & \sigma_{n 2} \\
\vdots & \vdots & \ddots & \vdots \\
\sigma_{1 n} & \sigma_{2 n} & \cdots & -\sum_{j=1}^{n-1} \sigma_{n j}
\end{array}\right]\left[\begin{array}{c}
p_{1}(t) \\
p_{2}(t) \\
\vdots \\
p_{n}(t)
\end{array}\right]=\left[\begin{array}{c}
\dot{p}_{1}(t) \\
\dot{p}_{2}(t) \\
\vdots \\
p_{n}(t)
\end{array}\right]
$$

where $p_{i}(t)$ is the probability of the $i$ th state at time instant $t$. The summation of all probabilities $p$ should be equal to 1 , and their derivatives $\dot{p}(t)$ should be replaced with 0 in order to determine the steady state probabilities. Then, the set of equations can be solved simultaneously. The long-term probabilities are now in upper case notations. After substituting the last row of $Q, p$, and $\dot{p}$ with the summation of all the probabilities equal to 1, the corresponding matrix representation given in Equation (19) was obtained.

$$
\left[\begin{array}{cccc}
-\sum_{j=2}^{n} \sigma_{1 j} & \sigma_{21} & \cdots & \sigma_{n 1} \\
\sigma_{12} & -\sum_{\substack{j=1 \\
j \neq 2}}^{n} \sigma_{2 j} & \cdots & \sigma_{n 2} \\
\vdots & \vdots & \ddots & \vdots \\
1 & 1 & \cdots & 1
\end{array}\right]\left[\begin{array}{c}
P_{1} \\
P_{2} \\
\vdots \\
P_{n}
\end{array}\right]=\left[\begin{array}{c}
0 \\
0 \\
\vdots \\
1
\end{array}\right]
$$

The set of linear equations was solved to obtain the long-term probabilities for all states. Depending on the weather conditions, the system was classified as either an up or down state. A sum of long-term probabilities for each category was obtained, indicating the system's availability margins.

If $P_{1}, P_{2}, P_{3}$ and $P_{4}$ denote the steady state probabilities of states 1 to 4 respectively, the system's availability margins can be computed using (20).

Availability without MGTs $=P_{1}+P_{2}$

Unavailability without MGTs $=1-P 1-P 2$

Availability with MGTs $=P 3$

Availability without MGTs $=P 4$

In the subsequent sections, these values will be evaluated and compared with the ones obtained using MCS. 


\section{Results and Case Studies}

\subsection{Synopsis of Base Case Parameters}

In this work, all reliability studies were performed on the RBTS. The power mix portfolio of generation types (including WTG, PV, and MGT) utilized in the reliability assessment are presented in Table 5. It was assumed to supply a residential load profile characterized by the maximum peak demand of $13 \mathrm{~kW}$.

Table 5. System configurations for the base case study.

\begin{tabular}{cccc}
\hline \hline No. PVs & No. WTs & No. MGTs & No. Bat. \\
\hline 10 & 10 & 10 & 1 \\
\hline \hline
\end{tabular}

First, MCS is applied to evaluate the RBTS reliability with/without the conventional backup.

\subsection{MCS Results}

The simulations were performed for a 50-year period to obtain more accurate values, as described in [9]. The results pertaining to the base case with no conventional backup are shown in Table 6 .

Table 6. Reliability assessment results without backup units.

\begin{tabular}{cccc}
\hline \hline Availability & LOEE & LOLE \\
$(\mathrm{kWh} / \mathrm{Yr})$ & 6560120.215 & 1206.5 & $\begin{array}{c}\text { AENSI } \\
(\mathrm{Wh} / \mathrm{int} .)\end{array}$ \\
\hline 0.8646 & 65633.917 \\
\hline \hline
\end{tabular}

Table 7 indicates that integrating backup DGs within the system results in increased reliability levels.

Table 7. Reliability assessment results with MGT Units.

\begin{tabular}{cccc}
\hline \hline Availability & $\begin{array}{c}\text { LOEE } \\
(\mathrm{kWh} / \mathrm{Yr})\end{array}$ & $\begin{array}{c}\text { LOLE } \\
(\mathrm{h} / \mathrm{Yr})\end{array}$ & $\begin{array}{c}\text { DGSI } \\
(\mathrm{W} / \mathrm{Wh})\end{array}$ \\
\hline 0.99664 & 305004.965 & 29.94 & 1.8116 \\
\hline \hline
\end{tabular}

The load duration curve (LDC) in Figure 6 can be used when selecting the most optimal number of backup units. The minimum or base load in Figure 6 suggests that at least four MGTs would be required to improve the system reliability. It can also be deduced from the LDC because the peak demand durations throughout the year are usually short, having 10 MGTs would likely be sufficient to significantly enhance the system availability. The DGSI and LOEE indices were used to obtain the annual amount of backup power produced by MGTs. Such data would likely be required for cost analysis studies.

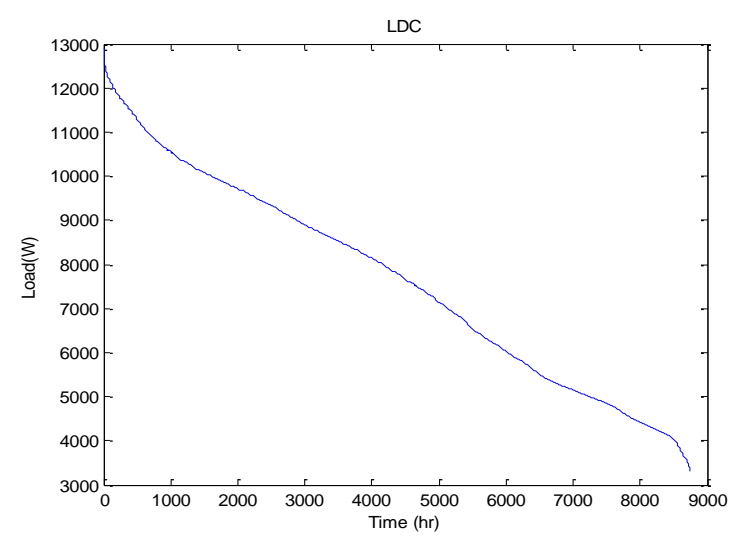

Figure 6. LDC of a single residential load. 


\subsection{Markov Modeling Results}

Using the results reported in the previous section, the occurrences of the four Markov states defined in Section 4 were detected. These are presented in the $\sigma$-matrix below.

$$
\sigma=\left[\begin{array}{cccc}
0 & 0.4391 & 0.0623 & 0.0015 \\
0.4452 & 0 & 0.0546 & 0.0007 \\
0.3760 & 0.0193 & 0 & 0.0142 \\
0.2236 & 0 & 0.6664 & 0
\end{array}\right]
$$

Then, the $Q$-matrix in (22) was developed.

$$
Q=\left[\begin{array}{cccc}
-0.5028 & 0.4452 & 0.3760 & 0.2236 \\
0.4391 & -0.5005 & 0.0193 & 0 \\
0.0623 & 0.0546 & -0.4095 & 0.6664 \\
0.0015 & 0.0007 & 0.0142 & -0.890
\end{array}\right]
$$

Using the Q-matrix, the system of Markov equations (23) was formed.

$$
\left[\begin{array}{cccc}
-0.5028 & 0.4452 & 0.3760 & 0.2236 \\
0.4391 & -0.5005 & 0.0193 & 0 \\
0.0623 & 0.0546 & -0.4095 & 0.6664 \\
1 & 1 & 1 & 1
\end{array}\right]\left[\begin{array}{l}
P_{1} \\
P_{2} \\
P_{3} \\
P_{4}
\end{array}\right]=\left[\begin{array}{l}
0 \\
0 \\
0 \\
1
\end{array}\right]
$$

Solving this system of linear equations yields the following steady state probabilities:

$$
\left[\begin{array}{l}
P_{1} \\
P_{2} \\
P_{3} \\
P_{4}
\end{array}\right]=\left[\begin{array}{c}
0.4594 \\
0.408 \\
0.1295 \\
0.0031
\end{array}\right]
$$

According to the availability margins defined in section 7, the system's availability margins were calculated, as shown in Table 8.

Table 8. Reliability assessment results using Markov modeling.

\begin{tabular}{cccc}
\hline \hline Availability without MGTs & Unavailability without MGTs & Availability with MGTs & Unavailability with MGTs \\
\hline 0.8674 & 0.1326 & 0.9969 & 0.0031 \\
\hline \hline
\end{tabular}

The values reported in Table 9 below indicate that the results obtained using the developed Markov model were very close to those obtained using SMCS.

Table 9. Absolute percentage error between the results yielded by SCMS and those obtained using Markov modeling.

\begin{tabular}{ccc}
\hline \hline $\begin{array}{c}\text { Availability without MGTs } \\
(\%)\end{array}$ & Unavailability without MGTs (\%) & Availability with MGTs (\%) \\
\hline 0.324 & 2.068 & 0.0261 \\
\hline \hline
\end{tabular}

\subsection{Sensitivity Analysis}

A sensitivity analysis was performed to investigate the effects of variations in the size of different parameters of the generation and storage systems on the system availability. The analysis was applied to the base case described in the previous section, whereby the number of WTs, PV panels, batteries, and MGTs was changed one component at a time.

Figure 7 below shows the effects of varying the size of the wind energy intake of the standalone system by changing the number of integrated WTs. In this case, system availability was evaluated assuming absence 
of conventional backup. As can be seen from the histogram, when only one WT was integrated, the system became unavailable. This case highlights the importance of wind energy, as the maximum power generated from a single WT used in this study was greater than that of a single PV panel. Similarly, if 10 WTs were integrated, the system's availability would be equivalent to that of the base case (Tables 6 and 8).

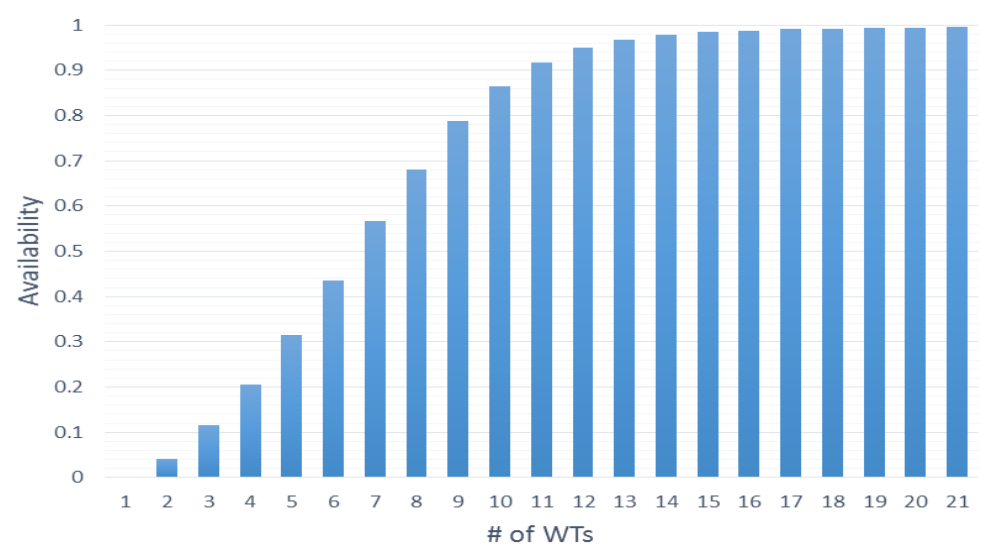

Figure 7. Availability sensitivity of a system without MGTs to the number of integrated WTs.

A similar sensitivity study was performed on the PV generation system, and the results are depicted in Figure 8. As expected, the system's availability was low when a single panel was utilized. However, the availability was equivalent to $55 \%$ as compared to $0 \%$ in Figure. 6 . This confirms the importance of wind in maintaining the desired level of availability. Once again, when 10 panels were integrated, the base case availability was reached.

When increasing the storage capacity of the base case, the same availability level was obtained. Interestingly, adding one more battery markedly increased system availability. However, the effect of increasing the storage size became saturated as the number of batteries increased, mainly due to not having significant amounts of renewable energy to be stored.

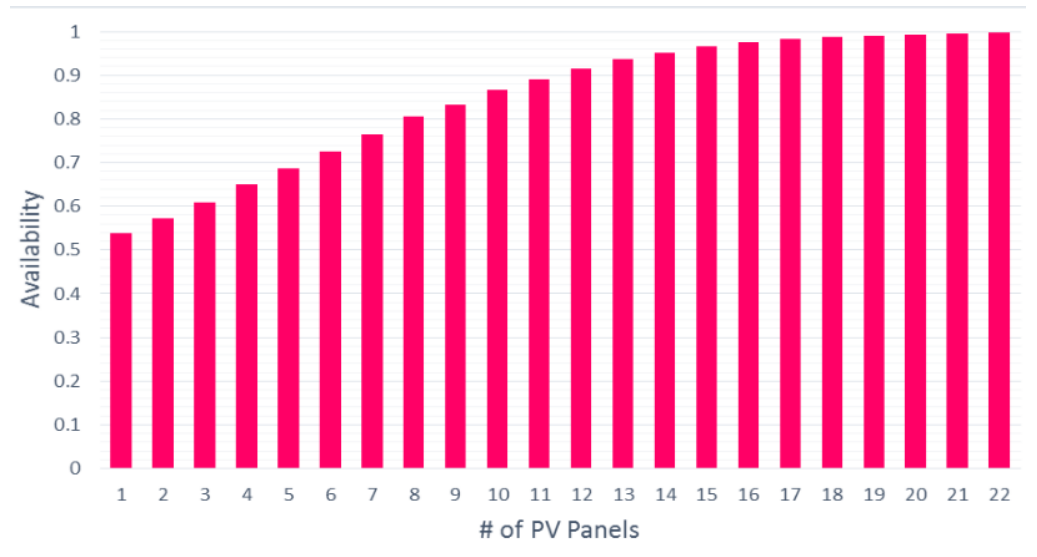

Figure 8. Availability sensitivity of a system without MGTs to the number of integrated PV panels.

Figure 9 illustrates the effect of variations in the size of the conventional energy source of the system backup when renewable energy and storage cannot satisfy the demand. System reliability initially remained almost unchanged. This outcome was anticipated as the base load was higher in those cases (Figure 6), making them impractical. The base case availability was achieved using 10 MGTs. It can be assumed that this is the most appropriate size, since the effects of increasing the number of MGTs further on system reliability became negligible. 


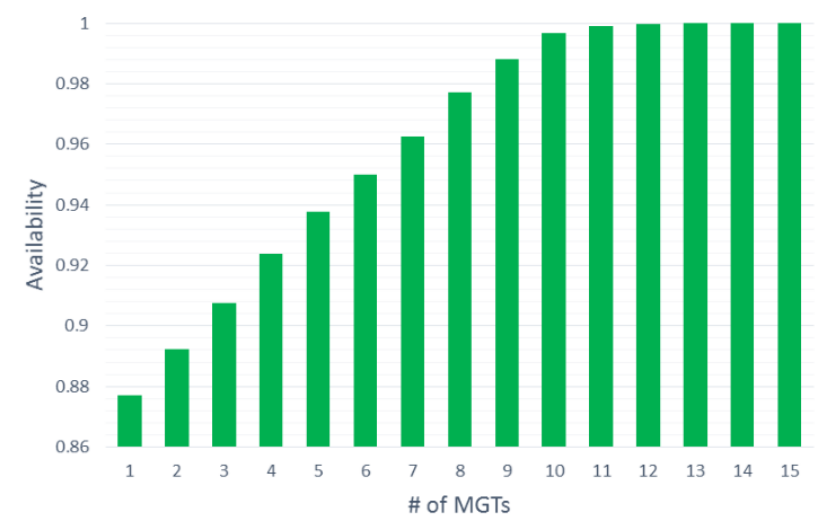

Figure 9. Availability sensitivity of a system with conventional backup to the number of MGTs.

\section{Conclusion}

A standalone microgrid consisting of renewable energy resources backed up by conventional MGTs was studied. Each generator model was designed and implemented on the IEEE RBTS load system. A reliability assessment of the standalone system was conducted using SMCS and Markov modeling. The system's availability margins, computed from the developed Markov model, were verified by comparing them with the values obtained using SMCS. Green energy sources were found to be unreliable relative to other types of conventional DGs mainly due to their direct dependence on stochastic weather factors. However, utilizing energy storage devices increased the system reliability. In addition, the effects of variations in the sizing of all system generation and storage components were investigated.

\section{Acknowledgment}

The authors acknowledge the support of the King Fahd University of Petroleum and Minerals (KFUPM), Dhahran, Saudi Arabia.

\section{References}

[1] Ghahderijani, M., Barakati, S., and Tavakoli, S., "Reliability evaluation of stand-alone hybrid microgrid using sequential Monte Carlo simulation," 2012 Second Iranian Conference on Renewable Energy and Distributed Generation (ICREDG), pp. 33-38, 6-8 March 2012.

[2] Zhou, W., Lou, C., Li, Z., Lu, L., and Yang, H., "Current status of research on optimum sizing of standalone hybrid solar-wind power generation systems," Proc. Applied Energy, vol. 87, pp. 380-389, 2010.

[3] Billinton, R. and Jonnavithula, A., "Application of sequential Monte Carlo simulation to evaluation of distributions of composite system indices," in Proc. 1997 IEE Generation, Transmission and Distribution conf., pp. 87-90.

[4] Fang, L., and Cai, J., "Reliability assessment of microgrid using sequential Monte Carlo simulation," Journal of Electronic Science and Technology, vol. X, no. X, pp. 31-34, 2011.

[5] Billinton, R. and Allan, R., Reliability Evaluation of Power Systems, Plenum Press, New York, 1996.

[6] Allan, R., Billinton, R., Sjariej, I., Goel, L., and So, K., "Reliability test system for education purposes Basic distribution system dates and results," IEEE Transactions on Power Systems, vol. 6, no. 2, pp. XX-YY, 1991.

[7] Mancasi, M., and Vatu, R., "Smart grids reliability indices assessment using sequential Monte Carlo method," in Environment and Electrical Engineering (EEEIC), 2015 IEEE 15th International Conference on, vol., no., pp. 2066-2071, 10-13 June 2015.

[8] Kahrobaee, S., Asgarpoor, S., and Kahrobaee, M., "Optimum renewable generation capacities in a microgrid using generation adequacy study," in T\&D Conference and Exposition, 2014 IEEE PES, vol., no., pp.1-5, 14-17 April 2014.

[9] Saleem, A., Banat, I., and AlMuhaini, M., "Reliability assessment of a stand-alone hybrid system using Monte Carlo simulation," 2016 13th International Multi-Conference on Systems, Signals \& Devices (SSD), Leipzig, Germany, 2016, pp. 714-719. 